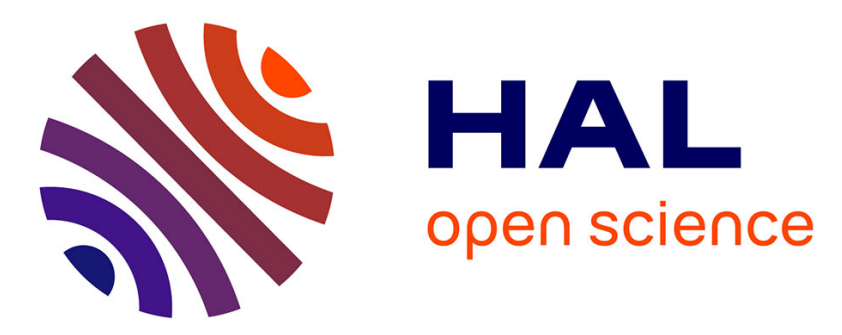

\title{
Simulation numérique du plissement des tôles lors de leur transport en continu dans les usines sidérurgiques
}

\author{
Nicolas Jacques, Akli Elias, Michel Potier-Ferry, Hamid Zahrouni
}

\section{To cite this version:}

Nicolas Jacques, Akli Elias, Michel Potier-Ferry, Hamid Zahrouni. Simulation numérique du plissement des tôles lors de leur transport en continu dans les usines sidérurgiques. 7e colloque national en calcul des structures, CSMA, May 2005, Giens, France. hal-01812964

\section{HAL Id: hal-01812964 \\ https://hal.science/hal-01812964}

Submitted on 12 Jun 2018

HAL is a multi-disciplinary open access archive for the deposit and dissemination of scientific research documents, whether they are published or not. The documents may come from teaching and research institutions in France or abroad, or from public or private research centers.
L'archive ouverte pluridisciplinaire HAL, est destinée au dépôt et à la diffusion de documents scientifiques de niveau recherche, publiés ou non, émanant des établissements d'enseignement et de recherche français ou étrangers, des laboratoires publics ou privés. 


\title{
Simulation numérique du plissement des tôles lors de leur transport en continu dans les usines sidérurgiques
}

\author{
Nicolas Jacques*,** — Akli Elias** — Michel Potier-Ferry* — \\ Hamid Zahrouni* \\ * Laboratoire de Physique et Mécanique des Matériaux (LPMM), UMR CNRS 7554 \\ Université de Metz, Ile du Saulcy, F-57045 Metz cedex 01 \\ \{jacqueni, potier-ferry,zahrouni\}@lpmm.univ-metz.fr \\ ** ARCELOR Research SA \\ Voie Romaine, F-57280 Maizières-lès-Metz \\ akli.elias@irsid.arcelor.com
}

\begin{abstract}
RÉSUMÉ. Cet article porte sur l'étude de la formation de défauts de forme, appelés plis, lors du transport en continu de bandes métalliques. Un modèle éléments finis a été mis au point pour simuler le passage d'une bande sur un rouleau. Il nous permet de préciser les mécanismes à l'origine du plissement. La traction appliquée à la bande afin de la guider, engendre l'apparition de contraintes compressives secondaires faisant flamber la bande. Nous avons observé que ces ondulations sont fortement accentuées lors du mouvement de la tôle. Ce phénomène est lié au frottement bande-rouleau. Dans certains cas, les plis se forment par l'action d'un mécanisme cumulatif plastique.

ABSTRACT. The development of plastic creases, called wrinkles, during sheet metal conveying in processing lines is considered. Finite element analyses are carried out to modelize wrinkling when a sheet is passing over a roll. A description of this phenomenon is proposed. When a strip is stretched, small compressive stresses occur near the roll and cause the strip buckling. Simulations have shown that friction between sheet and roll accentuates the postbuckling patterns during strip displacement. In some cases, buckles evolve to create a wrinkle by a plastic cumulative mechanism.

MOTS-CLÉS : plissement, tôles minces, transport de bandes, (post-)flambement, éléments finis. KEYWORDS: wrinkling, sheet metal, web handling, (post-)buckling, finite element..
\end{abstract}

Nom de la revue. Volume $\mathrm{X}-\mathrm{n}^{\circ} \mathrm{X} / 2001$, pages 1 à $\mathrm{X}$ 


\section{Introduction}

La fabrication de produits plats en acier nécessite de nombreuses étapes, une fois laminées les tôles doivent encore subir divers traitements (recuit, galvanisation, " coating »...) avant d'être commercialisables. Lors de ces procédés, les tôles sont soudées entre elles et se présentent sous forme d'une bande de longueur plus ou moins infinie, elles sont transportées à l'aide de rouleaux, on parle de transport en continu. Un des principaux problèmes rencontrés lors de ce transport est la formation de plis. Les plis sont des défauts de forme qui apparaissent lors du passage de la tôle sur les rouleaux. Ils se présentent sous la forme d'une ondulation plastique fortement marquée et généralement orientée selon la longueur de la tôle. La présence de plis rend les tôles impropres à la commercialisation. La compréhension et la maîtrise de ce phénomène apparaissent comme un enjeu important pour les sidérurgistes.

Un phénomène précurseur au plissement est le flambement de la bande, il est dû à la traction appliquée afin de guider la bande. Les tôles étant relativement larges et minces, les tractions appliquées dans les usines sont supérieures aux tractions critiques de flambage. Cependant, cette traction induit un effet stabilisant, l'amplitude des ondulations reste modérée, même si la traction est nettement supérieure à la traction critique de flambement. La limite élastique est rarement atteinte. La formation de plis ne peut donc pas être expliquée par un flambage plastique. En fait, l'action de mécanismes dissipatifs conduit à la modification du mode de flambage lors du déplacement de la tôle. Les plis apparaissent lorsque le niveau de traction atteint une valeur appelée traction critique de formation des plis (TCFP) beaucoup plus élevée que la traction critique de flambement (fig. 1-a).

A notre connaissance, il y a assez peu de travaux publiés concernant ce phénomène. Les principales études proviennent des sidérurgistes japonais (Sasaki et al., 1984), (Matoba et al., 1994), (Kaseda et al., 1994). Néanmoins ces études, relativement anciennes, ne proposent pas une description complète des phénomènes à l'origine du plissement. Plus récemment, Valérie Gueydan (1997) a proposé une étude numérique du flambement des tôles sous traction dans les lignes de recuit continu. Ces travaux mettent en évidence que la principale cause du flambement est le profil des rouleaux utilisés dans les usines. En effet, afin d'améliorer le guidage de bande, les rouleaux ne sont pas cylindriques, mais légèrement convexes (fig. 1-b). Cette géométrie conduit, lors de l'application de la traction, à l'apparition de contraintes compressives secondaires, qui peuvent faire flamber la bande. Le modèle proposé par Gueydan ne simule pas le mouvement de la bande, il ne permet donc pas d'étudier la formation des plis.

\section{Modélisation par éléments finis}

Un modèle élément fini a été réalisé à l'aide du code ABAQUS. Il prend en compte un rouleau et une portion de tôle. Le rouleau est modélisé à l'aide d'une 
surface rigide. Nous avons considéré un profil de rouleau conique, le rouleau est constitué d'une partie centrale cylindrique, appelée table, prolongée par deux cônes (fig. 1-b). Il s'agit d'un des profils les plus utilisés dans les usines. La tôle est modélisée avec des éléments de coque mince (S8R5, éléments quadratiques à intégration réduite). Le contact unilatéral avec frottement de Coulomb est défini entre la tôle et le rouleau. Nous utilisons une loi de comportement élasto-plastique avec écrouissage isotrope (modèle de Prandl-Reuss). Les simulations sont réalisées en statique non-linéaire. Les calculs sont effectués en deux étapes : on applique une traction à la tôle, puis on simule le déplacement de la tôle combinée à la rotation du rouleau, la traction restant constante (fig. 2).

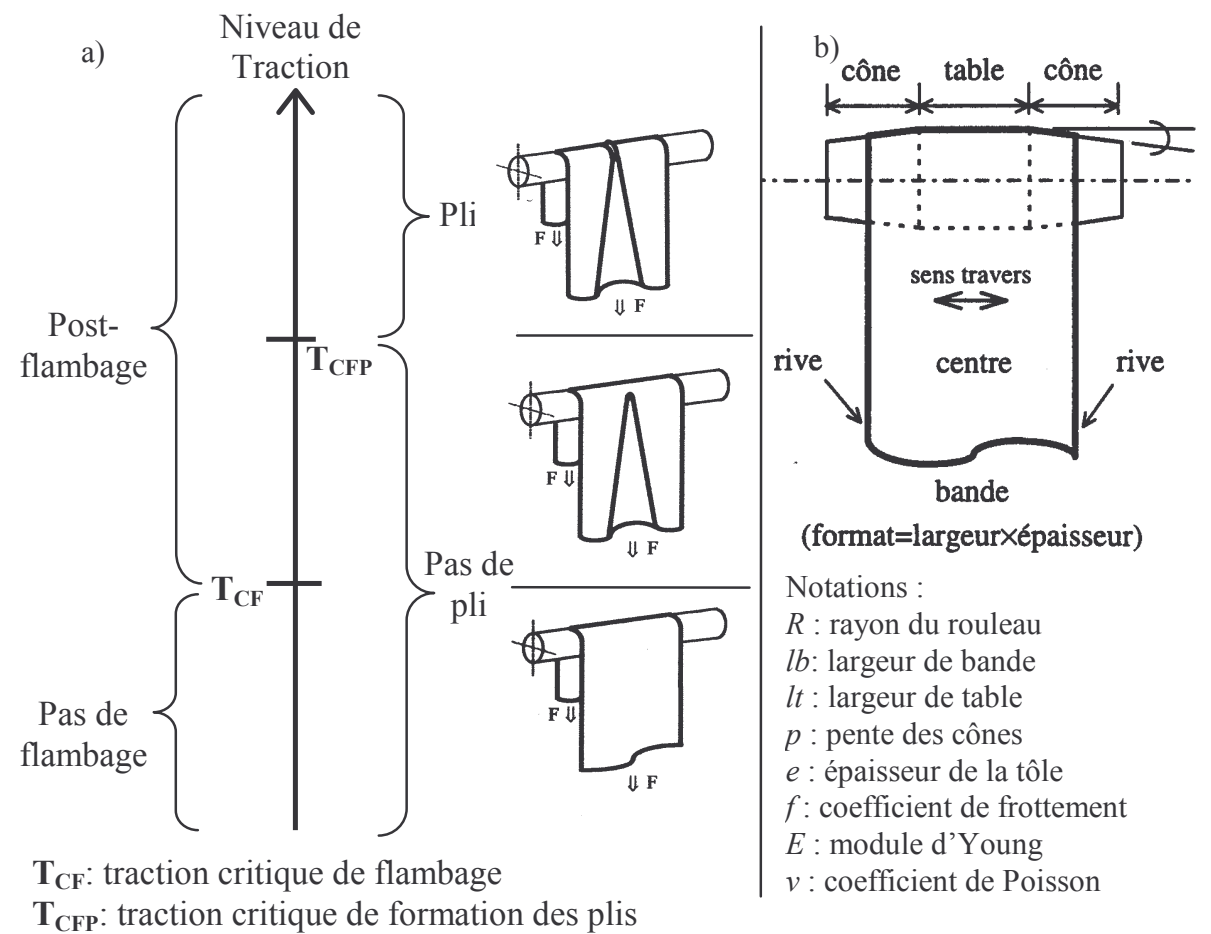

Figure 1. a) Notions de flambement et de plissement. b) Illustration des profils de rouleau coniques.

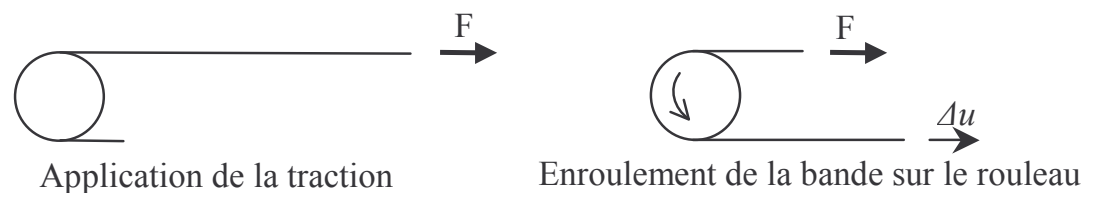

Figure 2. Les deux étapes des simulations de transport de bande 


\section{Flambement sous traction de la bande}

Pour certaines structures, l'application d'un chargement de traction engendre l'apparition de contraintes compressives secondaires pouvant causer le flambement de la bande. Des exemples sont présentés dans (Yoshida et al., 1981) et (Friedl et al., 2000). Dans notre cas, ce phénomène est lié au profil de rouleau. Lorsque l'on applique la traction, le contact s'établi tout d'abord sur la partie cylindrique du rouleau, ce qui crée une inhomogénéité des contraintes de traction au niveau du rouleau à l'origine de contraintes compressives dans le sens travers. Ces contraintes sont localisées à proximité du rouleau. Le flambement se présente sous forme d'ondulations très allongées s'estompant progressivement avec la distance du rouleau (image de droite de la figure 4).

\section{Evolution des ondulations de flambement lors du déplacement de la bande}

\subsection{Les contraintes compressives augmentent lors de l'enroulement}

Comme je l'ai déjà indiqué, la traction induit un effet stabilisant sur les ondulations. Par conséquent, même dans le post-flambement lointain, l'amplitude des ondulations reste modérées. Ce flambement génère donc peu de contraintes supplémentaires et est généralement élastique. Cependant, les simulations montrent une forte accentuation des ondulations lors du mouvement de la bande. En fait, lorsque la bande flambée arrive sur le rouleau, il existe un léger glissement transversal entre la tôle et le rouleau qui engendre des efforts de frottement. Par conséquent, des contraintes compressives supplémentaires apparaissent lors de l'enroulement. La figure 3 montre que ces contraintes peuvent avoir un niveau supérieur à celles dues à la traction. Ce sont ces contraintes supplémentaires qui provoquent l'importante accentuation des ondulations de flambement.

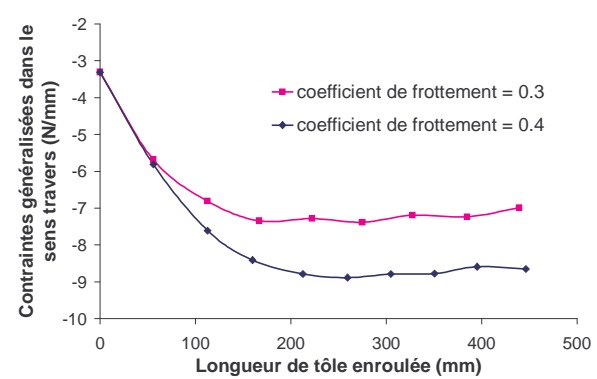

Figure 3. Augmentation des contraintes compressives dans le sens travers lors de l'enroulement d'une bande élastique pour deux valeurs du coefficient de frottement. $R=220 \mathrm{~mm}, l b=1000 \mathrm{~mm}, \mathrm{lt}=400 \mathrm{~mm}, p=1.33 \mathrm{~mm} / \mathrm{m}, e=0.2 \mathrm{~mm}, E=70000 \mathrm{MPa}$, $v=0.3$, traction appliquée $25 \mathrm{MPa}$. 


\subsection{Le pli se forme par l'action d'un mécanisme cumulatif plastique}

L'augmentation des contraintes de flexion durant la phase d'accentuation due au frottement peut permettre d'atteindre la limite élastique. Dans certains cas, ces déformations plastiques conduisent à la mise en œuvre d'un mécanisme cumulatif plastique : si le flambage a marqué plastiquement la tôle, des ondulations résiduelles se propagent avec le mouvement de la bande. L'enroulement de ces ondulations sur le rouleau crée des contraintes additionnelles qui accentuent le flambage en amont. Par conséquent, des ondulations plus marquées arrivent sur le rouleau, accentuant encore davantage le flambement en amont, un cycle auto-catalytique se met en place. Le pli se forme progressivement sur le rouleau au fur et à mesure qu'il se propage avec le défilement de la bande (fig. 4). Ce phénomène est mis en évidence par la figure 5-a présentant l'évolution de la courbure des ondulations lors de l'enroulement pour différentes valeurs de la limite élastique. Au début, les courbes sont confondues. Durant cette phase d'accentuation due au frottement, l'augmentation de la courbure est environ proportionnelle à celle des contraintes compressives. Par la suite, les courbes se séparent. Pour les limites élastiques les plus faibles $(\leq 85 \mathrm{MPa})$, la courbure se met à augmenter très fortement après la première plastification, cela correspond à l'action du mécanisme cumulatif plastique, qui conduit à la formation d'un pli. La figure 5-b montre l'évolution de la courbure pour différents niveaux de tractions. Ces courbes sont très similaires à celles de la figure 5-a ; il semble donc que la TCFP, ici comprise entre 25 et $27.5 \mathrm{MPa}$, soit le niveau de traction engendrant l'action du mécanisme cumulatif plastique.

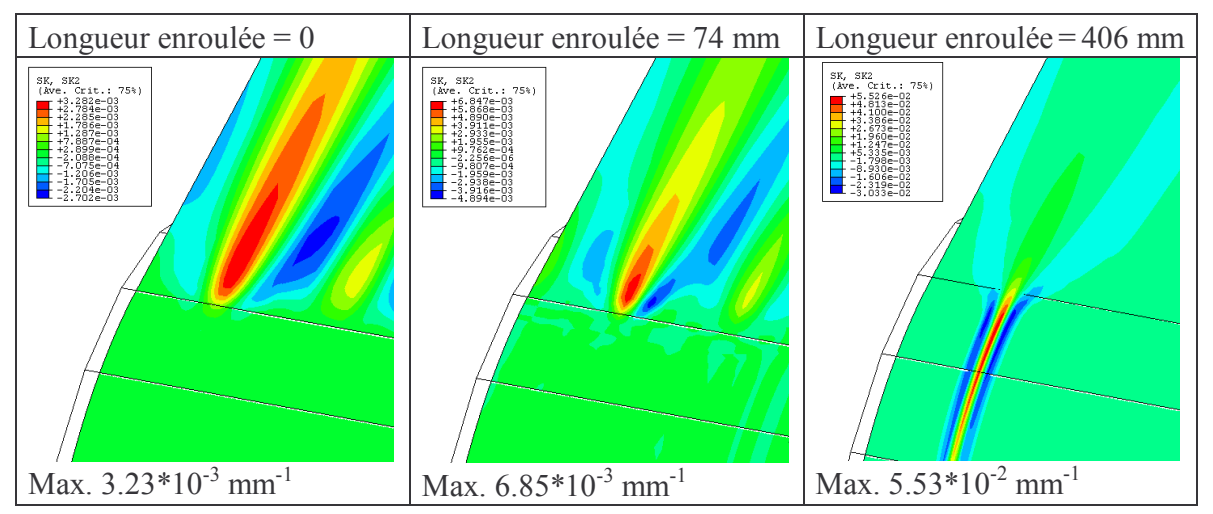

Figure 4. Formation d'un pli lors du déplacement de la bande. Ces figures montrent la portion de tôle arrivant sur le rouleau. Par symétrie, une seule moitié de la bande est maillée, l'arrête visible sur les images correspond au centre de la bande. Après un enroulement de $74 \mathrm{~mm}$, on observe une importante accentuation des ondulations due au frottement, la limite élastique est atteinte à ce moment. Le pli se forme ensuite par l'action du mécanisme cumulatif plastique. La tôle a un comportement elasto-plastique, la courbe d'écrouissage provient d'un essai de traction sur un aluminium, $f=0.4$, traction appliquée $30 \mathrm{MPa}$, autres données : cf. fig. 3. 

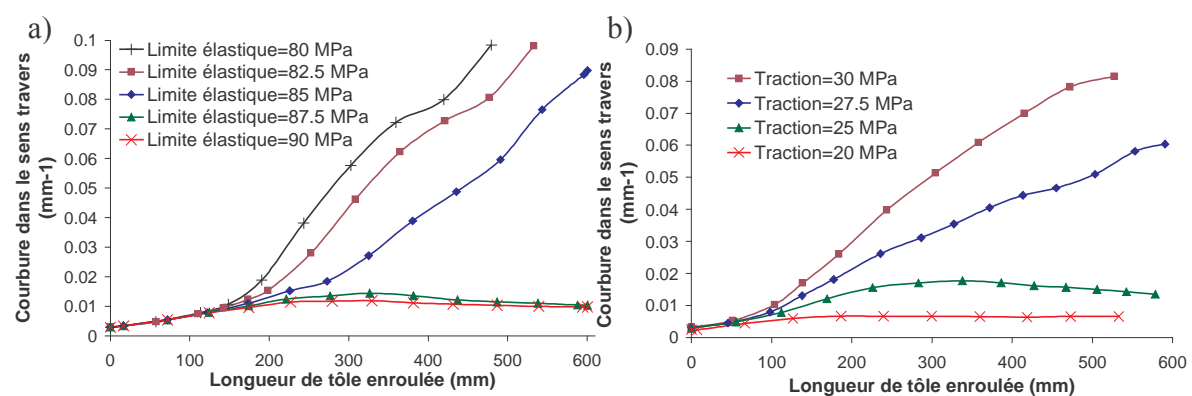

Figure 5. Evolution de la courbure des ondulations lors de l'enroulement. a) pour différentes valeurs de la limite élastique (comportement parfaitement plastique, $E=70000 \mathrm{MPa}, v=0.3)$, traction $25 \mathrm{MPa}$. b) pour différents niveaux de tractions. Dans les deux cas, les données non-précisées sont identiques à celles de la fig. 4.

\section{Conclusions}

Des simulations numériques nous permettent de comprendre la formations de plis lors du transport de bandes. Les rôles du frottement et de la plasticité sont mis en évidence. La modélisation proposée permet en outre d'évaluer la TCFP dans une configuration donnée et constitue donc un outil précieux d'aide à la conception des lignes continues.

\section{Bibliographie}

Sasaki T., Yangishima F., Hira T., Shimoyama Y., Abe H., Tahara K., Control of strip buckling and snaking in continuous annealing furnace, Kawasaki Steel Technical Report No. 9, 1984, p. 36-46.

Matoba T., Effect of roll crown on heat buckling and strip walk in continuous annealing lines, CAMP ISIJ vol. 5, 1994, p. 1459-1462.

Kaseda Y., Masui T., Control of buckling and crossbow in strip processing lines, Iron and Steel Engineer, September 1994, p. 14-20.

Gueydan V., Modélisation numérique du flambage de bandes en acier dans les recuits continus, Thèse de Doctorat de l'Université de Metz, 1997.

Yoshida K., Hayashi J., Hirata M., Yoshida buckling test, IDDRG, 1981, Kyoto, Japan.

Friedl N., Rammerstorfer F.G., Fischer F.D., Buckling of stretched strips, Computers and Structures, vol. 78, 2000, p. 185-190. 